\title{
Central Nervous System Non-Hodgkin Lymphoma
}

National Cancer Institute

\section{Source}

National Cancer Institute. Central Nervous System Non-Hodgkin Lymphoma. NCI

Thesaurus. Code C114779.

A non-Hodgkin lymphoma that arises from the central nervous system. 